\title{
Tardive Dyskinesia Outcomes: Clinical and Pharmacologic Correlates of Remission and Persistence
}

\author{
Roberto Cavallaro, M.D., Maria Grazia Regazzetti, M.D., Emanuela Mundo, M.D., \\ Viviana Brancato, M.D., and Enrico Smeraldi, M.D.
}

Roersible tardive dyskinesia (TD) outcomes have been reported in long-term neuroleptic (NL)-treated patients. In this study the course of TD outcomes was followed-up for 3 years in a population of 125 institutionalized schizophrenic patients (mean age 57.8 years) receiving continuous NL treatment. Tardive dyskinesia occurrence and severity were assessed by means of the Rockland Simpson Scale (RSS). The prevalence of TD rose from $39.2 \%$ at the first examination to $52.8 \%$ at last follow-up examination; however, $28.6 \%$ of TD-affected patients recovered and $30 \%$ improved. Significant risk factors for a persistent TD outcome result included age over 56 years, duration of illness over 30 years, and a total RSS score over 22. Cumulative NL exposure, over $3550 \mathrm{~g}$ of chloropromazine equivalents, was also a significant risk factor for TD. Results from this study confirm that there is the possibility of improvement and remission in an aged, long-term institutionalized population of $T D$ patients. In this report we point out prognostic factors for positive outcome. [Neuropsychopharmacology 8:233239, 1993]
KFY WORDS: Drug-induced dyskinesia; Schizophrenia; Prognosis; Risk factors; Tranquilizing agents; Age

Tardive dyskinesia (TD) has always been considered the most dramatic side effect of chronic neuroleptic (NL) treatment, mainly because of its widely assumed irreversibility. This assumption has influenced the approach to long-term treatment of schizophrenia and related disorders in dyskinetic patients because of concerns about iatrogenesis. A number of cases showing resolution or improvement of TD symptoms have been reported since the first findings about TD were published in the late fifties. The early reports focused on patients whose NL treatment was withdrawn (Uhr-

From the Department of Neuropsychiatric Sciences, S. Raffaele Hospital, University of Milan, Italy.

Address correspondence to: Dr. Roberto Cavallaro, Department of Neuropsychiatric Sciences, S. Raffaele Hospital, via Prinetti, 29, mo Milan, Italy.

Received November 27, 1991; revised May 14, 1992 and August 3. 1992; accepted August 10, 1992. brand and Faurbye 1960; Paulson 1968; Crane 1973; Hershon et al. 1972; Jeste et al. 1979; Glazer et al. 1984, 1990), whereas more recently and more frequently, new findings about remissions of TD symptomatology in patients receiving continuous NL treatment have been published (Casey 1985; 1991; Casey et al. 1986; Casey and Gardos 1990; Gardos et al. 1988; Bergen et al. 1989; Morgenstern et al. 1987; Glazer et al. 1991).

Data from prospective, retrospective, longitudinal, and descriptive studies reveal that in patients receiving long-term NL therapy, TD often has a reversible course. These data also identify some factors that seem to be associated with remission or amelioration of symptomatology. Among the variables studied, those most frequently involved were a younger age, NL dosage at the first recognition of TD, length of NL treatment, and severity of illness. Recently, Glazer et al. (1991) identified age and the presence of nonorofacial dyskinetic movements at first evaluation as predictors of persistent TD.

The best way to correctly evaluate the risk/benefit 
ratio of continuing NL treatment in the natural history of TD is to undertake longitudinal studies with repeated measures (Gardos and Cole 1983), but problems may arise from population mobility. Elderly populations have been considered the most suitable for short-term longitudinal study (Hoffmann et al. 1989).

The aim of this study was to evaluate the course of TD in a population of schizophrenic patients receiving continuous NL treatment, so as to identify changes in severity, remissions, new cases, and their possible predictive indicators.

\section{METHODS}

Data were obtained from a population of chronic schizophrenics residing in a psychiatric hospital. Residents of this kind of institution are those who have decided to remain, after the passage of a 1978 law that prohibits new admissions to long-term psychiatric hospitals. For this reason, the population we observed was peculiar in that they had only two possible ways to leave their community, discharge or death.

Initially 148 patients were admitted to the study for the first observation (Altamura et al. 1990); however, the population available for both observations, thereby fulfilling the aim of the study, was reduced to 125 patients ( 70 males and 55 females with a mean age of 57.8 years) because 23 patients died. To be included, patients had to be diagnosed as suffering from chronic schizophrenia or a schizoaffective disorder, and to have been treated with NL drugs for at least 3 months prior to entry into the study. To establish diagnoses, charts were reviewed and the diagnoses made on the basis of clinical presentation and course, in accordance to DSM III criteria. Exclusion criteria were concomitant or past neurologic diseases, alcoholism, or leucolobotomy.

Patients were rated separately by two trained psychiatrists by means of the Rockland Simpson Scale (RSS), abbreviated version for TD (Simpson et al. 1979). Scores were calculated by averaging the two ratings in a conservative way (half scores were corrected to the lower digit). Raters were blind to the patients' drug history and current pharmacologic treatment. Clinical charts of the patients admitted to the study were reviewed for age, age in relation to diagnosis onset, and duration of illness. For a group of 60 patients, whose missing charts did not reach a cumulative period of more than 6 months, NL and anticholinergic treatments were recorded. Neuroleptic and anticholinergic drug doses were transformed into chloropromazine and benzotropine equivalents, respectively.

Daily doses and cumulative exposure to the drugs under study were calculated. Information about drug holidays and electroconvulsive shock therapy (ECT) treatments were obtained from the same charts. A di- agnosis of TD was made with a minimum total score of 19 on the RSS (in employing the rating scale, 17 is the negative score) which could be reached with a score of 3 in at least one item or a score of 2 in two items in separate body areas. Patients who amassed this score in the latter way were assessed again after 3 weeks and a score definitively assigned (only patients with at least one " 3 " score at the second evaluation with no change in daily NL dose were considered positive). Since the RSS is a very sensitive and specific instrument for rating TD in comparison with other scales, and since our diagnostic criteria were conservative, we think that this way of evaluating TD, if performed by well-trained raters, can also be useful and reliable in identifying those diffuse mild signs of TD which are often the first, underevaluated signs of this disease. In this view, we think that our criteria are comparable to those of Schooler and Kane, but fitted to the rating scale used. Patients also were assessed for extrapyramidal side effects (EPS) by means of the Simpson and Angus rating scale (SARS).

\section{Statistical Analysis}

Statistical analysis was performed using analysis of variance (ANOVA) and the multifactor ANOVA to compare parametric variables; cross-tabulation with the Fisher exact test for contingency tables was used to calculate relative risks. Only evaluations of patients who were available at both observations were considered for the statistical analysis. Patients were evaluated at admission (time 0 ) and after 3 years (time 1 ) by the same raters (R.C. and M.G.R.) using the same criteria with the same concordance in ratings (correlation coeffcient $>0.90$ for both observations; correlation analysis text).

\section{RESULTS}

The sample studied consisted of 125 patients, 70 males and 55 females. The TD prevalence figure changed from $39.2 \%$ at first observation to $52.8 \%$ at last follow-up examination at the end of the observation period. Among males, prevalence increased from $42.9 \%$ to $51.4 \%$, whereas among females prevalence rose from $34.5 \%$ to $54.5 \%$; mean age was significantly higher $(p<0.001)$ in females $(61.8$ years $\pm 9.6 \mathrm{SE})$ than in males ( 54.0 years $10 \mathrm{SE}$ ). Of the 125 patients reassessed after 3 years, 45 remained without TD (no TD in the two evaluations, called "negative TD"), 31 developed TD (TD diagnosis at second evaluation only, called "new TD"), 35 were still affected (TD diagnosis in both evaluations, called "persistent TD"), and 14 had a complete remission of TD symptomatology (TD diagnosis at first evaluation only, called "remitted TD"). Table 1 shows the clinical and demographic characteristics of the whole sample and of the patients with a complete pharmacologic his- 


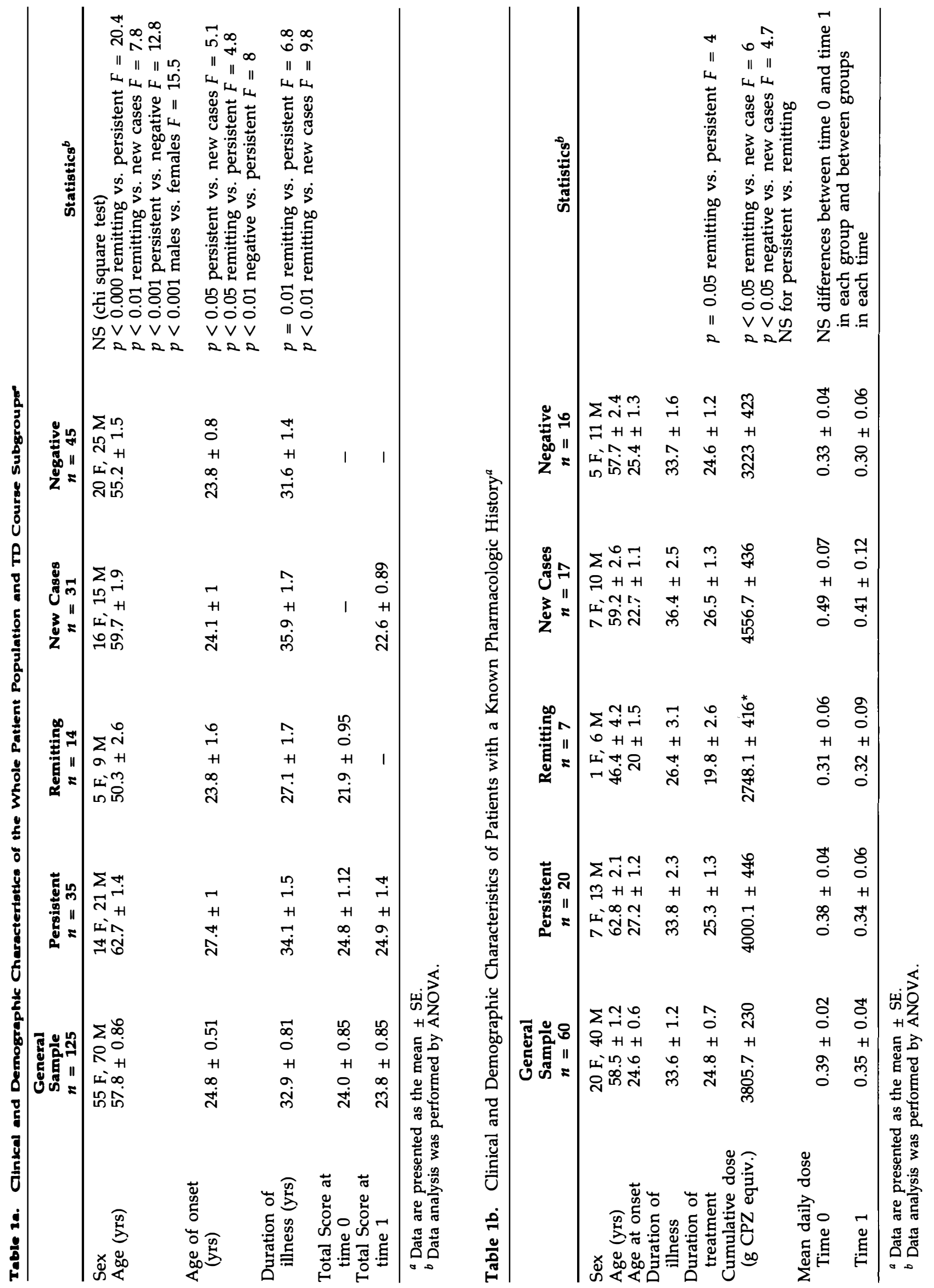


tory and relative statistics. At the second evaluation, the 49 patients originally diagnosed as affected by TD showed the following four patterns of disease course: 13 patients $(26.5 \%)$ worsened, $7(14.8 \%)$ were unchanged, $15(30 \%)$ had a decrease in severity, and 14 (28.6\%) recovered.

Although we considered all possible conditions (Table 1), data analysis was oriented toward possible differences in remission and persistence. The mean age, duration of illness, and age at onset of psychiatric illness were significantly lower $(p<0.0000, p=0.01, p<0.05$, respectively) in the remitted TD group, and no significant sex effect was found (Table 1). As a consequence of this finding, the relative risks of developing persistent TD were studied for age and duration of illness. These two variables seemed to be the most representative for studying the possible neuropathologic and biologic implications of drug treatment, institutionalization, and the natural course of schizophrenia in TD outcome. From this viewpoint, however, age at onset did not give any additional information. The relative risk of developing persistent TD for patients aged more than 56 years (the median value of the variable considered in the entire population) at time 0 , was 9.1 ( $p<$ 0.005 two-tailed Fisher's exact test). The relative risk of developing persistent TD for patients with a duration of illness of more than 30 years (median value) was 11.5 $(p<0.005)$.

Considering the SARS total scores, the risk of developing persistent $T D$ for patients with a score higher than 22 at first evaluation (time 0 ) was 5.5 times higher $(p<0.05)$ than in patients with a lower score. Among patients with persistent TD, total scores remained unchanged in 7 , were lower in 15 , and higher in 13 compared to basal evaluation. Among these patients, the improved group had a lower mean age and duration of illness than the unchanged and worsened groups (Table 2). During the 3 years of collection, the relative risk for developing TD in patients aged over 56 years varied from 2.3 (time 0 ) to 3.8 (time 1) with a new higher significance ( $p<0.0001$, Fisher's exact two-tailed test). At first evaluation, EPS were found in $12.8 \%$ of the total sample. The highest rate was found in the negative TD group (22.2\%), followed by a rate about one-half as high $(11.4 \%)$ in the persistent TD group. Extrapyramidal side effects were present among $7.1 \%$ of remitting patients and $3.2 \%$ of new patients. At the second evaluation, EPS were present in $11.2 \%$ of all patients, with rates of $20 \%, 8.6 \%, 7.1 \%$, and $3.2 \%$, respectively (following the same outcome order).

Total EPS scores were not correlated to total TD scores in either of the two evaluations (correlation analysis) nor was the presence of EPS at first observation related to outcome at 3 years (cross-tabulation, Fisher's exact test). In the group of patients whose pharmaco- logic history was collected, $33.3 \%$ were affected by persistent TD, $11.7 \%$ by remitting TD; $28.3 \%$ were new cases, and $26.7 \%$ were still not affected. A total of $25.9 \%$ of the originally TD-affected patients recovered (Table 1). No significant differences were found in the variables considered between this group and the general sample. No significant difference in mean daily NL doses was found between groups and between times for each group. No difference in mean cumulative anticholinergic doses between groups and between two observations was found (5699.36 mg benzotropine equivalents at baseline and 5748.05 at second evaluation). No difference was found between outcome groups in history of anticholinergic treatments, before the first observation or in the period between the two observations.

Duration of NL treatment ranged from 8 to 16 years in seven patients, from 17 to 24 years in 17 patients, from 25 to 32 years in 32 patients, and from 33 to 40 years in 4 patients. A statistically significant difference was found in mean treatment duration between the persistent and remitting groups $(p=0.05)$ (Table 1).

Mean cumulative doses of NL drugs for patients with persistent TD were higher than in patients with remitting TD, but did not reach statistical significance (Table 1), and relative risk, calculated for a cumulative NL dose higher than $3550 \mathrm{~g}$, had a result of 9 . No statistically significant difference was found between groups relative to percentages of patients with increased, unchanged, or decreased absolute daily doses (Table 3 ).

Considering the presence of TD in our population at second the evaluation, a significant difference $(p<$ 0.01 ) in mean cumulative doses (in grams) of NLs was found between affected $(4257.5 \pm 312 \mathrm{SE})$ and nonaffected patients ( $3078 \pm 318 \mathrm{SE})$. This result was affected by duration of treatment, as shown by the MANOVA test in which the same variables covariated by duration of treatment and were different at $p=0.05$. Relative risk for occurrence of TD calculated on a "cutoff" value (median value) of mean cumulative NL dose (3550 g) was $3.7(p<0.05)$.

In patients with persistent TD, nine presented with reduction (absolute decrease of scale score), six with worsening of symptomatology (absolute increase of score), and five were unchanged (no change in score). Among these patients, the improved group had a lower mean cumulative dose of NL drugs than the unchanged group, and the latter group's variables were lower than the worsened patients (Table 2). No statistically signifcant difference was found in daily doses between improved, unchanged, and worsened patients (ANOVA) and no correlation was found between change in daily doses and change in total TD scores (regression analysis). Mean daily doses did not differ significantly in these groups at time 0 and time 1 . No difference was 


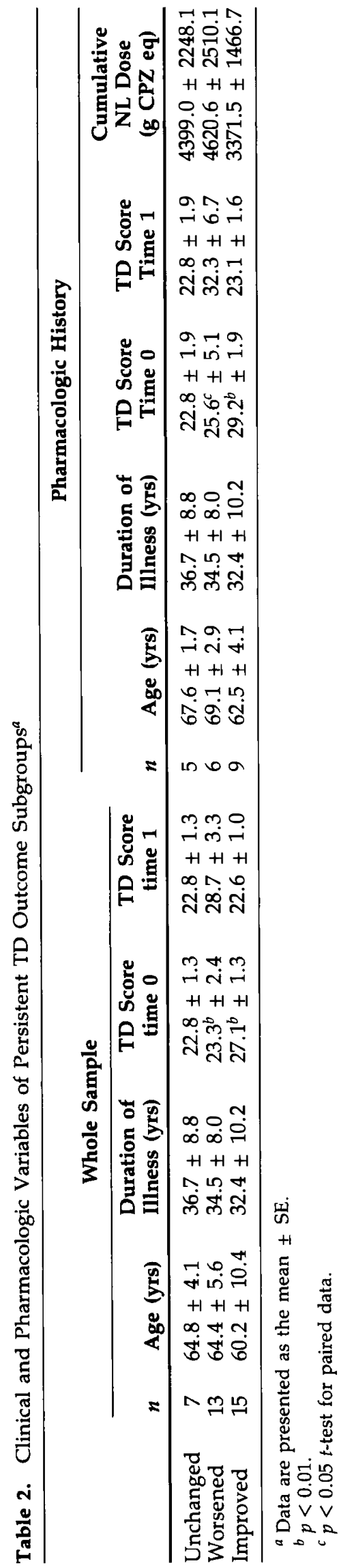

found between groups (both outcome groups and TDprevalence groups) if previous ECT treatments and number and length of drug holidays were considered.

\section{DISCUSSION}

The occurrence of TD places significant weight on the pharmacologic management of schizophrenia. For this reason, reports on the short-, medium-, and long-term outcome of TD have become more and more frequent in the international literature. Recent observations point out that populations of aged patients are the most suitable for short-term studies on TD (Hoffmann et al. 1989).

Because of its clinical and demographic characteristics, our population seemed to be particularly well suited for studying the course of TD. In fact, the diagnoses were homogeneous, the populationlacked mobility, and institutionalization exposed all patients to the same extrapharmacologic variables (i.e., socio-rehabilitative treatments as well as the institutionalization effects themselves). Further pharmacologic treatment was completely recordable for a large and representative subgroup.

The prevalence of TD increased from the time of the first evaluation. This is almost certainly an effect of increasing age, but could also be due to the continued administration of NL drugs, or both. This last hypothesis seems to be the more probable. A clear influence of the duration of treatment, which could be considered a time-dependent toxicologic effect, wasfound (see MANOVA results), but this does not completely account for the effect of differences in cumulative doses of NL drugs, which is a direct measure of total quantitative exposure. In fact, after statistical analysis of the duration of treatment as a covariate, its significance is reduced, but not lost. If we consider this finding along with that of no significant difference found in NL cumulative doses at the first evaluation (Altamura et al. 1990) (difference was at the $p=0.08$ level) 3 years ago, we can hypothesize that a threshold of quantitative exposure to NL was overcome in several patients during these 3 years, with the concomitant weight of a timedependent variable (increased duration of treatment together with aging of neurologic substratum).

After 3 years, the prevalence value was $20 \%$ higher in females (versus a $7.5 \%$ increase in males). This result is consistent with that of Woerner et al. (1991) who found a similar pattern of prevalence and prevalence differences according to sex, with a greater prevalence among older females. Considering that a significant number of these patients were postmenopausal, these data seem to confirm the hypothesis of a protective role of estrogens suggested by some of the literature (Glazer et al. 1985; Smith et al. 1978). 
Table 3. Cross-Tabulation of Dose Changes by Outcome $e^{a}$

\begin{tabular}{lccccc}
\hline & Persistent & Remitting & New Cases & Negative & Row Total \\
\hline Decreased & $12(60 \%)$ & $4(57.1 \%)$ & $11(64.7 \%)$ & $10(62.5 \%)$ & $37(61.7 \%)$ \\
Unchanged & $0(0 \%)$ & $0(0 \%)$ & $1(5.9 \%)$ & $0(0 \%)$ & $1(1.7 \%)$ \\
Increased & $8(40 \%)$ & $3(42.9 \%)$ & $5(29.4 \%)$ & $6(37.5 \%)$ & $\frac{22(36.7 \%)}{60(100 \%)}$ \\
Column total & $20(33.3 \%)$ & $7(11.7 \%)$ & $17(29.4 \%)$ & $16(37.5 \%)$ & 60 \\
\hline
\end{tabular}

${ }^{a}$ Chi square and Fisher's exact test, NS.

In our sample, the outcome of the originally TDdiagnosed patients was quite positive, with $30 \%$ of patients improved and $28 \%$ completely recovered. The rate of this last more favorable course is relatively high. This finding of no certain irreversibility of TD in patients receiving continuous NL treatment confrms previously published reports (Casey and Gardos 1990; Baldessarini et al. 1990). Furthermore, as in our study, patients with improving or remitting TD in comparable studies were younger and had less severe TD (Yassa et al. 1984; Seeman 1981). It is interesting to note from other studies that possible influences of dose variations on TD presentation (covert TD becoming overt because of reduction of NL dose and vice versa) should not significantly influence our data. In fact, mean daily doses were not significantly different between the two evaluations (time 0 and time 1 ) or between groups, although a general decrease in daily doses was found. When increases/decreases in individual doses were studied, no significant differences were found among groups. Thus, there is a possibility that the decrease in mean daily NL doses, even if not statistically significant, could have influenced scores, but this should have had a minor effect and only in particular patients.

Risk factors found for a poor outcome appear to be the same as those for TD occurrence. Age, duration of illness, and duration of treatment were significantly higher in the persistent TD group than in the remitting group. A direct implication of this result may be that more factors depending on the neurologic substratum (aging), toxicological charge (duration of treatment), and duration of psychiatric illness (but this could also be due to a "tracking" effect of the variable duration of treatment) come together, not only to determine TD occurrence, but also its outcome. From this viewpoint, a possible genetic predisposition to TD would not become phenotypically evident if some complex conditions were not realized and maintained. We think it is also worth noting that in our descriptive analysis regarding persistent TD courses (statistical analysis was not performed due to the small number in each group) the same variables were found to be lower in patients with an improved course.

In any case, data from this study are subject to the possibility that TD can have an "intermittent" course with remissions and exacerbations, as was found by certain authors who performed repeated observations through long-term studies (Glazer et al. 1991; Bergen et al. 1989). This question can be answered only after further follow-up of the population currently being investigated. Repeated observation may give, if the "intermittent" course is also found in our population, additional information on the course of TD.

We believe that the further study of a neurotoxicologic model in which the genetic predisposition to TD expresses itself in a range of different, but related clinical manifestations and courses, could be of paramount importance in the management of long-term pharmacologic treatment of schizophrenia and in the development of new pharmacologic strategies.

\section{REFERENCES}

Altamura AC, Cavallaro R, RegazzettiMG (1990): Prevalence and risk factors for tardive dyskinesia: A study in an Italian population of chronic schizophrenics. Eur Arch Psychiatry Clin Neurosci 240:9-12

Baldessarini RJ, Cole JO, Davis JM, Gardos G, Preskorn SH, Simpson GM, Tarsy D (1980): Tardive dyskinesia: A Task Force Report of the American Psychiatric Association, Washington, DC, American Psychiatric Association, PP 43-66

Bergen JA, Eyland EA, Campbell JA, Jenkings P, Kellehear K, Richards A, Beaumont PJV (1989): The course of tardive dyskinesia in patients on long-term neuroleptics. Br J Psychiatry 154:523-528

Casey DE (1985): Tardive dyskinesia: Reversible and irreversible. In Casey DE, Chase T, Christensen AV, Gerlach J (eds), Dyskinesia: Research and Treatment. Berlin, Springer, pp 88-97

Casey DE (1991): Tardive dyskinesia. Long-term outcome. Biol Psychiatry 29:11S-144S

Casey DE, Gardos G (1990): Tardive dyskinesia: Outcome at 10 years. Schizophrenia Res 3:1-11

Casey DE, Povlsen UJ, Meidahl B, Gerlach J (1986): Neuroleptic-induced tardive dyskinesia and parkinsonism: Changes during several years of continuing treatment. Psychopharmacol Bull, 22:250-253

Crane GE (1973): Persistent dyskinesia. Br J Psychiatry 122:395-405

Gardos G, Cole J (1983): The prognosis of tardive dyskinesia. J Clin Psychiatry 44:177-179 
Gardos G, Perenyi A, Cole JO, Samu I, Kocsis E, Casey DE (1988): Seven-year follow-up of tardive dyskinesia in Hungarian outpatients. Neuropsychopharmacology 1:169-172

Glazer WM, Moore DC, Schooler N, Brenner LM, Morgenstern $\mathrm{H}$ (1984): Tardive dyskinesia: A discontinuation study. Arch Gen Psychiatry 41:623-627

Glazer WM, Naftolin F, Morgenstern H, Barnea ER, MacLusky NJ, Brenner LM (1985): Estrogen replacement and tardive dyskinesia. Psychoneuroendocrinology 10: 345-350

Glazer WM, Morgenstern H, Schooler N, Berkman CS, Moore DC (1990): Predictors of improvement in tardive dyskinesia following discontinuation of neuroleptic medication. Br J Psychiatry 157:585-592

GlazerWM, Morgenstern H, Doucette JT (1991): The prediction of chronic persistent versus intermittent tardive dyskinesia. Br J Psychiatry 158:822-828

Hershon HI, Kennedy PF, McGuire RJ (1972): Persistence of extrapyramidal disorders and psychiatric relapse after withdrawal of long-term phenothiazine therapy. Br J Psychiatry 120:41-50

Hoffmann WF, Ballard LC, Casey DE (1989): Longitudinal assessment of older schizophrenic patients. Am J Psychiatry 146:403-404

Jeste DV, Potkin SG, Sinha S, Feder S, Wyatt RJ (1979):
Tardive dyskinesia. Reversible and irreversible. Arch Gen Psychiatry 36:585-590

Morgenstern H, Glazer WM, Gibowski LD, Holmberg S (1987): Predictors of tardive dyskinesia: results of a crosssectional study in an outpatient population. J Chron Dis 40:319-327

Paulson CW (1968): An evaluation of the permanence of the "tardive dyskinesia." Nerv Syst 24:692-694

Seeman MV (1981): Tardive dyskinesia: Two-year recovery. Comp Psychiatry 22:189-192

Simpson GM, Lee JH, Zoubok B, Gardos G (1979): A rating scale for tardive dyskinesia. Psychopharmacology 64: 171-179

Smith JM, Oswald WT, Kucharski T, Waterman LJ (1978): Tardive dyskinesia, age and sex differences in hospitalized schizophrenics. Psychopharmacology 58:207-211

Uhrbrand L, Faurbye A (1960): Reversible and irreversible dyskinesia after treatment with perphenazine, chloropromazine, reserpine, and electroconvulsive therapy. Psychopharmacologia 1:408-418

Woerner MG, Kane JM, Lieberman JA, Alvir J, Bregmann KJ, Borenstein M, Schooler NR, Mukherjee S, Rotrosen J, Rubinstein M, Basavaraju N (1991): The prevalence of tardive dyskinesia. J Clin Psychopharmacol 11:34-42

Yassa R, Nair V, Schwartz G (1984): Tardive dyskinesia: A two-year follow-up study. Psychosomatics 25:852-855 